\title{
Development and Evaluation of Evidence Based Patient Education Booklet in Hindi Language for Prevention of Shoulder Pain in Paraplegia
}

\author{
Taniya Verma ${ }^{1}$, Chitra Kataria ${ }^{2}$ \\ ${ }^{1}$ Research student, MPT Musculoskeletal, ISIC-Institute of Rehabilitation Sciences, \\ Vasant Kunj, New Delhi, India \\ ${ }^{2} \mathrm{Ph} . \mathrm{D}$ in Physiotherapy and Rehabilitation, Chief of Rehabilitation Services, \\ ISIC-Institute of Rehabilitation Sciences- New Delhi
}

Corresponding Author: Chitra Kataria

\begin{abstract}
Background: Shoulder pain is one of the most frequent complaints of Paraplegia with a prevalence reported to range from $50 \%$ to $60 \%$. It has been attributed to the increase in upper extremity (UE) weight bearing following lower extremity paralysis.

Purpose of the study: To develop and Evaluate an Evidence Based Patient Education Booklet in Hindi Language for Prevention of Shoulder Pain in Paraplegia along with pictorial presentation.

Design: A methodological study

Method: It consist of 4 steps. 1) Literature review and informal interview of 2 doctors, 7 physiotherapists, 5 occupational therapists and 10 patients were used to determine the domains of the education booklet. 2) Development of content of the evidence based patient education booklet according to domains found in step 1 and develop a rough draft of booklet in Hindi Language. 3) Evaluation of the evidence based booklet by expert panel using SAM and to modify the booklet to produce the final booklet draft. 4) Pilot study on patients to take their views regarding the booklet developed.

Results: The domains found in step 1 were: Education about pain, Education about the treatment options, Ergonomics modifications, Positioning, Wheel chair selection and transfer techniques, Exercises, General precautions. The content was developed in step 2. In step 3 the SAM score is- $88.13 \%$ and final booklet was produced after modification. In step 4 the patient rated the booklet easy to read and understand with an overall rating of 9/10
\end{abstract}

Conclusion: The developed patient education booklet came out to be easy to read and understand and of superior quality according to Suitability assessment of material questionnaire and hence should be made a part of patient education.

Key words: Patient education booklet, Shoulder pain in paraplegia, Suitability assessment of material questionnaire, booklet in Hindi language, Pain prevention and management, Health education material

\section{INTRODUCTION}

Shoulder pain is one of the most frequent complaint in paraplegic patients with a prevalence range from $30 \%$ to $60 \%$ $(1,2)$. Paraplegic patients use assistance of devices for mobilization which includes wheelchair, cane, crutches These patients have repetitive overuse of upper limbs as a weight bearing extremity Since an extremity meant for mobility is exclusively used for weight bearing purpose, the problem of shoulder pain in such population is quite common. (3)

Health education provided to the patients and family care givers can help reduce morbidity and mortality of long term disease. Patient education can be delivered by various methods, out of which booklets have been advocated as it is a simple, cost effective, and popular method of providing health-related information to patients. It also 
enhances understanding retention and application of the information (4) Language is one of the barrier in delivering the patient education.(5)

A booklet developed for patient education is more helpful if evaluated with suitable measures available such as suitability assessment of materials, readability scales, questionnaire and checklist about the content of education material which have been used in various studies. (6-8) It is also important to obtain feedback from the target audience on their preference on the design, content and delivery of written health information. Pilot study are done for evaluation of patient education booklet in which comment regarding the booklet is taken from patients. $(8,9)$ In this study, a patient education booklet will be developed in Hindi language for prevention of shoulder pain in paraplegia and would be evaluated by doctors, physiotherapist, occupational therapist followed by a pilot study.

\section{MATERIALS AND METHOD Design}

Step1-Determination of domains of the evidence based patient education booklet for prevention of shoulder pain on the basis of literature review and patient inputs via informal interview.

Step2-To develop the content of the evidence based patient education booklet according to domains found in step 1 and develop a rough draft of booklet in Hindi Language.

Step3-Evaluation of the evidence based booklet by expert panel using SAM and to modify the booklet to produce the final booklet draft.

Step4-Pilot study on patients for evaluation of evidence based patient education booklet for prevention of shoulder pain in paraplegia in Hindi language

\section{Sample population}

Inclusion Criteria: Doctors involve in treating paraplegic patients for at least a period of 2 years. Physiotherapist and
Occupational therapist having a certificate of Delhi council of physiotherapy and occupational therapy or is a member of Indian Association of physiotherapy and is involve in treating paraplegic patients for at least a period of 2 years. Paraplegic Patients with Age: 18-60 years with complete/ incomplete spinal cord injury (T1 to T12 Level) (more than 1 month) who is a Manual wheel chair user. (Traumatic and atraumatic both) and Who read and Understand Hindi Language. Expert in Hindi language with B.A and M.A in hindi language and experience of 10 years or more as a teacher/professor in school or college.

Exclusion Criteria: Absence of consent for participation. Patients who are diagnosed with other neurological condition (egdementia, seizures,) uncontrolled cardiovascular disease, severe respiratory distress and who are Unable to understand and read Hindi Language

\section{Intervention}

Literature review was done first after which informal interview was framed and were asked from 2 doctors, 7 physiotherapist, 5 occupational therapist and 10 patients. During literature review, A search strategy with the following words was done on Google scholar, the Cochrane library, PubMed/Medline and Science direct: Patient education, Patient information, Shoulder pain prevention in Paraplegia, wheelchair users shoulder pain etc. Relevant articles with full text were included in the study

After explaining the study, consent was taken from the expert panel and patients for their participation in the study.

\section{Informal Interview}

The purpose of this interview was to determine which areas should be included in the patient education booklet, according to them. It comprises of a few open-ended and closed ended questions that were developed following the literature review outlined earlier in relation to the patient education booklet's areas, as well as discussions with 
Taniya Verma et.al. Development and evaluation of evidence based patient education booklet in Hindi Language for prevention of shoulder pain in paraplegia

the research guide. During the interview, it took about 15-20 minutes.

Various articles were referred to determine the content and dosage of the domains. The images used in demonstrations for the exercises were clicked and graphically converted by graphic designer. Technical medical terminologies used in research articles were made easy by the researcher and a rough booklet was developed in Hindi language. This was done with the constant interaction of the researcher and the research guide. A rough draft of booklet was thus developed. After the development of evidence based patient education booklet, it was evaluated for its readability and suitability. Since no scale is developed so far to evaluate the readability of Hindi text, 2 Hindi experts were given the copy of booklet for their suggestions and the correction was done in booklet accordingly. The suitability assessment of material questionnaire was filled by doctors, physiotherapists, and occupational therapist after reading the instructions to use it. lastly Pilot study was done on patients to take their views regarding the booklet developed.

\section{Data analysis}

Descriptive statistics were used to summarize the demographics of the physiotherapists, occupational therapists, doctors and patients.

\section{RESULTS}

\section{Result of step 1:}

Table 1 and table 2 shows the domain obtained by literature review and informal interview respectively.

Table 1: Domains obtained from literature review.

\begin{tabular}{|l|}
\hline Ergonomics modification \\
\hline Positioning \\
\hline Wheelchair selection and adjustments \\
\hline Transfers techniques. \\
\hline Exercise \\
\hline Precautions \\
\hline
\end{tabular}

According to SAM questionnaire the booklet score obtained was $88.13 \%$ which rated it of superior quality. The average patient rating for the booklet came out to be 9 out of 10 during pilot study.
Table 2: Information regarding domains that were highlighted during informal interview

\begin{tabular}{|l|l|}
\hline \multicolumn{1}{|c|}{ Group } & \multicolumn{1}{|c|}{$\begin{array}{c}\text { Domain highlighted after the } \\
\text { informal interview }\end{array}$} \\
\hline Doctors & $\begin{array}{l}\text { Use of exercise } \\
\text { Transfer education } \\
\text { Activity modification } \\
\text { Pain management }\end{array}$ \\
\hline Physiotherapist & Use of exercise \\
& Proper wheelchair techniques \\
& Ergonomics modifications \\
& Pain management \\
\hline Occupational therapist & Use of exercise \\
& Proper wheelchair techniques \\
& Ergonomics modifications \\
& Pain management \\
& General precautions \\
\hline Patients & Pain related education \\
& Transfer education \\
& Activity modification \\
& Wheelchair related education \\
& Exercise education. \\
\hline
\end{tabular}

\section{DISCUSSION}

This study is designed to develop and evaluate a patient education booklet in Hindi language through a focus group feedback process for prevention of shoulder pain in paraplegia. Patient education programs aims to impart knowledge and skills to individuals so that they can manage their condition in a better manner.(5)

Determination of domain was an important step in the study as it provides the basic framework for the development of patient education booklet as here, we are trying to find out the domains. It also becomes easier for the reader to refer content in a booklet by presence of domains. Present study incorporates literature review along with informal interview given to physiotherapist, occupational therapists, doctors, and patients to determine the domain of the booklet which was not performed so extensively in any research revied during this step

In the 2nd step, best possible efforts were made to formulate the content of the domains. There was a conscious effort to simplify the material of these domains and provide appropriate visual representations to make it easier for patients to understand. The language has been simplified to the greatest extent possible for easy understanding of the patients.

After evaluation and modification of the booklet, the final booklet was drafted. 
The following information was also provided in the booklet: contact information for any suggestions, further readings, list of contents was added before the actual content of the booklet starts. The evidence based patient education booklet is fairly easy to read and understand and is of superior quality according to Suitability Assessment of Material questionnaire and therefore can be given to patients for the prevention of shoulder pain.

\section{CONCLUSION}

The evidence based patient education booklet is fairly easy to read and understand and is of superior quality according to Suitability Assessment of Material questionnaire and therefore can be given to patients for the prevention of shoulder pain.

\section{ACKNOWLEDGEMENT(S)}

The author acknowledges Dr. Chitra Kataria Chief of Rehabilitation Services, Principal, ISIC-IRS-Indian Spinal Injuries Centre, New Delhi along with other faculty members who helped me to complete my study. This research received no specific grant from any funding agency in the public, commercial, or not-for-profit sectors.

\section{Acknowledgement: None}

\section{Conflict of Interest: None}

\section{Source of Funding: None}

\section{REFERENCES}

1. Chhabra HS, Arora M. Demographic profile of traumatic spinal cord injuries admitted at Indian Spinal Injuries Centre with special emphasis on mode of injury: a retrospective study. Spinal Cord. 2012 Oct;50(10):74554.

2. Acharya BD, Rawal M, Rokaya PK, Karki D, Limbu D, Binaya PL. Prevalence of shoulder pain disability among assistive device users with paraplegia. Journal of Karnali Academy of Health Sciences. 2018 Oct 6;1(2):33-7.

3. Hastings J, Goldstein B. Paraplegia and the shoulder. Physical Medicine and Rehabilitation Clinics. 2004 Aug 1;15 (3):699-718.

4. Friedman AJ, Boyko S, Cosby R, Cancer Care Ontario. Patient Education Panel. Effective teaching strategies and methods of delivery for patient education. Cancer Care Ontario; 2013.

5. Van Wyk K, Backwell A, Townson A. A narrative literature review to direct spinal cord injury patient education programming. Topics in spinal cord injury rehabilitation. 2015;21(1):49-60.

6. Louw A, Butler DS, Diener I, Puentedura EJ. Development of a preoperative neuroscience educational program for patients with lumbar radiculopathy. American journal of physical medicine \& rehabilitation. 2013 May 1;92(5):446-52.

7. Morowatisharifabad MA, Yoshany N, Sharma M, Bahri N, Jambarsang S. Readability and suitability assessment of educational materials in promoting the quality of life for postmenopausal women. Przegląd Menopauzalny Menopause Review. 2020 Jul;19(2):80./

8. Nagpal M, Makhija M, Sachdev A. development and evaluation of evidence based pre-operative patient education booklet in lumbar Discectomy. Int $\mathrm{J}$ Physiother Res. 2014;2(1):359-64.

9. Oliveira SC, Lopes MV, Fernandes AF. Development and validation of an educational booklet for healthy eating during pregnancy1. Revista latinoamericana de enfermagem. 2014 Jul;22:61120.

How to cite this article: Verma T, Kataria C. Development and evaluation of evidence based patient education booklet in Hindi Language for prevention of shoulder pain in paraplegia. International Journal of Science \& Healthcare Research. 2021; 6(4): 166-169. DOI: https://doi.org/10.52403/ijshr.20211023 